Historia y comunicación social

ISSN-e 1988-3056

https://dx.doi.org/10.5209/hics.66291

\title{
Posverdad, fake-news y agenda política en el discurso de Trump en Twitter
}

\author{
Francisco Cabezuelo Lorenzo $^{1}$; Juan Luis Manfredi ${ }^{2}$
}

Recibido: 28 de enero de 2019. / Aceptado: 25 de septiembre de 2019.

Resumen. Este trabajo muestra los resultados del análisis de contenido de los 133 mensajes de Donald Trump en su perfil de Twitter (@realDonaldTrump) durante su primer mes como presidente electo de los Estados Unidos tras los comicios noviembre de 2016. Su análisis demuestra que Trump ha empleado Twitter de manera impulsiva y nada institucional para incrementar la afiliación partidista y manejar la opinión pública. Por un lado, mediante la exposición de ideas no convencionales sobre las relaciones internacionales de Estados Unidos. Y, por otro, porque el reflejo de los medios de comunicación internacionales refuerza el perfil heterodoxo del nuevo presidente. Ese posicionamiento refuerza la identidad del votante republicano medio con Trump en pleno debate sobre la posverdad y la desinformación en los nuevos contextos digitales.

Palabras clave: Twitter; Fake-News; Donald Trump; Neurocomunicación; Posverdad.

\section{[en] Post-Truth, fake-news and political agenda in Trump's speech on Twitter}

\begin{abstract}
This article shows the results of a detailed study using a content-analysis methodology of the 133 tweets published by President-Elect Donald Trump on Twitter (@realDonaldTrump) during his first month the presidential elections of November 2016. This study offers useful information about Trump's potential future lines of government, showing how Trump used Twitter to increase a potential party affiliation and manage public opinion. On one hand, Trump exposed unconventional ideas about foreign affairs. On the other hand, due to his image on the international media, Trump has reinforced his heterodox profile as a new president. Paradoxically, this position reinforces the self-identity of Republican voters with Trump in a context of debate about fake news and misinformation.
\end{abstract}

Keywords: Twitter; Fake-News; Donald Trump; Neuro-Communication; Post-Truth.

Sumario: 1. Introducción y estado de la cuestión; 2. Metodología: material y métodos; 3 . Análisis y resultados; 4 . Discusión y conclusiones; 5 . Referencias bibliográficas.

Cómo citar: Cabezuelo Lorenzo, F.; Manfredi, J. L. (2019). Posverdad, fake-news y agenda política en el discurso de Trump en Twitter, en Historia y comunicación social 24 (2), 449-483.

\footnotetext{
$1 \quad$ Universidad Complutense de Madrid.

fcabezue@ucm.es

2 Universidad de Castilla-La Mancha. juan.manfredi@uclm.es
} 


\section{Introducción y estado de la cuestión}

En lo que llevamos de siglo las aplicaciones de las neurociencias se han derivado a otros ámbitos científicos, especialmente a las ciencias sociales, incluyendo tanto las ciencias de la comunicación como la ciencia política y sociológica. La neurocomunicación ha sido propuesta como un nuevo paradigma en los estudios sobre comunicación, que aplica los avances y descubrimientos de la neurociencia a los procesos comunicativos (Gallardo-Paúls, 2016: 92). Destacan las investigaciones pioneras de António Damásio (1994) sobre la capacidad de las emociones para acceder y organizar la información, o las de George Lakoff (2009) en neurolenguaje y sus derivaciones en la acción política norteamericana. En España son pioneros los trabajos sobre la gestión del poder diluido y últimas formas de persuasión, de Jesús Timoteo Álvarez (2005 y 2007).

Las conclusiones a que las neurociencias y ciencias afines están llegando cambian en modo radical todo lo relativo al acceso de los individuos a la información y al conocimiento. Estos experimentos de vanguardia están lanzando conclusiones interesantes en lo relativo a la organización básica de la comunicación social, especialmente en el campo de la comunicación persuasiva, como demuestran los experimentos en torno al neuromarketing y neuroshopping, la relación entre cerebro, publicidad y opción de compra (Schreiber y Iacoboni, 2012) o sobre la neuropolítica o aplicaciones de las neuronas espejo al proceso de decisión de voto en Norteamérica (Schreiber, Fonzo, Simmons et al. 2013). Por su parte, Uña-Juárez, Fernández-Fernández \& Fernández-Camacho (2014: 132) añaden que la neurocomunicación permite estudiar cómo se procesa la información sensorial, lo que permite aplicar este conocimiento tanto a la creación de mensajes electorales y publicitarios como al diseño de estrategias para captar la atención del electorado y lograr altos niveles de impacto y memoria.

Las nuevas tendencias de investigación en comunicación se aplican también al estudio de un fenómeno de relevancia como es la aparición en las redes sociales e Internet, sobre todo de las fake news (en inglés), "noticias falsas" o también llamados "hechos alternativos" (en lengua española), realidades definidas también baj el término de posverdad (Macías-Varela, 2017). La utilización de la posverdad permite entender y describir la conmoción que han supuesto hechos como la victoria de Donald Trump o el Brexit, con una proliferación de noticias falsas en las redes de impredecibles consecuencias. Son ejemplos en los que la posverdad ha jugado un papel trascendental en la medida en que ha sobrepasado cualquier expectativa racional. La posverdad, por tanto, puede ser una mentira asumida como verdad o incluso una mentira reforzada como creencia o como hecho compartido en una sociedad a través de las diferentes herramientas digitales existentes en la actualidad.

De acuerdo con los posicionamientos tradicionales, nuestros cerebros y mentes están moldeados por nuestras experiencias, que tienen lugar principalmente en el contexto de la cultura en la que vivimos y nos hemos formado. En paralelo, los psicólogos no dejan de descubrir abundantes evidencias sobre la diversidad de la cognición humana y el comportamiento a través de los diferentes procesos culturales.

La posverdad se presenta como un híbrido bastante ambiguo cuyo significado que denota circunstancias en que los hechos objetivos influyen menos en la formación de la opinión pública, que los llamamientos a la emoción y a la creencia personal. 
Se trata de un "eufemismo de mentira llevada a cabo a través de las redes sociales e Internet", según Macías-Varela (2017).

Por otro lado, la cuestión de si los correlatos neurológicos de la cognición humana también dependen de la cultura no ha sido hasta ahora una cuestión tratada por los neurocientíficos. Sin embargo, recientes estudios transculturales han demostrado que el trasfondo cultural puede influir en la actividad neural que subyace a las funciones cognitivas de alto y bajo nivel. Estos hallazgos proporcionan un nuevo enfoque para distinguir los mecanismos neuronales de la cognición humana, sensibles a la cultura y invariantes a la cultura (Han \& Northoff, 2008: 646-654).

Entre ellas, la más importante es la tesis que considera que el entorno o contexto no es siempre una estructura impuesta desde el exterior sino una creación de los propios seres vivos o de cómo el modelo red manifiesta y expresa una inteligencia distribuida, una inteligencia a escamas (swarm intelligence) o inteligencia conectiva, con sus derivaciones neuronales en la medida en que el acto comunicativo no es una simple transferencia de mensajes sino una interacción de códigos con factores comunes (Timoteo, 2014: 43-68).

A pesar del status híbrido de Internet por sus complejas dificultades para su regulación, la red se ha presentado como reflejo y metáfora de la velocidad inherente de la era contemporánea. La irrupción de las Tecnologías de la Información y la Comunicación (de ahora en adelante TICS) ha permitido que los mensajes individuales se compartan, se hagan comunes a través de la redes sociales, convertidas en un fenómeno mundial. Los neurocientíficos están comenzando a capitalizar la ubicuidad del uso de los medios sociales para obtener nuevos conocimientos sobre los procesos cognitivos sociales, ya que las redes sociales proporcionan plataformas para que los usuarios satisfagan los impulsos sociales fundamentales, como conectarse con otros y gestionar la propia reputación con otros (Meshi, Tamir \& Heekeren, 2015: 771).

La sociedad en red se caracteriza porque aumenta el poder comunicativo de los ciudadanos y reconfigura su acceso a la información. El acceso a Internet aumenta en todo el mundo aumenta, con lo que también lo hace el número de usuarios de las redes sociales. La combinación de redes sociales, segundas pantallas y televisión ha propiciado la aparición de una nueva relación de los espectadores con la televisión en la que los habituales roles del paradigma de la comunicación se han alterado (Quintas Froufe \& González Neira, 2013: 83). El uso de las redes sociales es un fenómeno global con millones de usuarios en todo el mundo. Internet es el nuevo marco de desarrollo de la inteligencia colectiva (Timoteo 2014: 43). Las plataformas de redes sociales como Facebook o Twitter se han convertido debido a su popularidad e importancia no solo para incrementar o mantener relaciones interpersonales, sino también para llevar un registro de los acontecimientos de la vida pública, según Alvídrez \& Franco-Rodríguez (2016: 90), que estiman que en el caso de Twitter, su velocidad y alcance le han vuelto un instrumento habitual de la comunicación entre las personalidades públicas para atraer la atención de los usuarios. En este contexto, surge la posibilidad única de aprovechar la omnipresencia del uso de los medios sociales para obtener nuevos conocimientos sobre los procesos cognitivos sociales y los sistemas neuronales que los sustentan (Han \& Northoff, 2008: 646).

Las redes sociales, convertidas en los nuevos medios de comunicación propios de la sociedad digital, requieren modelos teóricos ajustados a un tipo de acciones comunicativas donde "no se intenta informar ni convencer sino sólo ocupar espacio 
público, conseguir la venta o el voto a cualquier precio", según Timoteo (2007: 360). A lo que Gallardo-Paúls (2016: 89) añade que el usuario de internet tiene una voluntad "casi exhibicionista" y el discurso de la red es "fundamentalmente, un discurso monológico y autocentrado, egocéntrico, que prescinde del destinatario". En este contexto digital, surge el nuevo desorden de la posverdad, que se caracteriza por la presencia de la mentira y de algunos de sus parientes como el fraude, la falsificación, el disimulo, el error, la ficción, la ironía, el secreto, la conspiración (Rodríguez Ferrándiz, 2018).

Las redes sociales actúan como motor de la inteligencia conectiva, un modo diferente de pensar y de relacionarse en la nueva sociedad en red. Gracias a las TICS, la red establece una conectividad simultánea y significativa entre múltiples usuarios, según los esquemas uno-varios, varios-uno o varios-varios (Uña-Juárez, Fernández-Fernández \& Fernández-Camacho, 2014: 132), porque impulsa la interactividad lúdica entre los contactos, porque sustituye la variable de la proximidad geográfica por aquella, propia del ciberespacio, donde la conexión se establece en función de intereses y de preferencias compartidos y porque procura acelerar la sinergia de los procesos de conocimiento descentralizado (Timoteo, 2014).

La creación de un corpus epistemológico se basa tanto en el conocimiento (hechos veraces, racionales) como en la percepción (hechos imaginados o supuestos) que construye el yo y el nosotros formado por la comunidad. Así, los sistemas neuronales que apoyan varios tipos de cognición social han sido establecidos por la investigación con comportamientos analógicos u offline. Sin embargo, estos sistemas neuronales también están involucrados en el uso de las redes sociales (Meshi, Tamir \& Heekeren, 2015: 771-782).

En este contexto surge también la posibilidad de aplicar la investigación en neurocomunicación al campo de la política (tanto nacional como internacional), la posverdad y el complejo mundo de las relaciones internacionales, donde cada vez las redes sociales ocupan un mayor protagonismo en lo que se ha venido a denominar la diplomacia cultural (Bound, Briggs, Holden \& Jones, 2007). En esta línea, autores como Rubio-Moraga y Sapag-Muñoz de la Peña (2014: 187-188) proponen identificar un área de conocimiento específica para la neuropolítica, concebida como una disciplina que investiga las bases biológicas (cerebrales) de las reacciones (mentales) que tienen los ciudadanos-votantes cuando son expuestos a los estímulos de la comunicación política, tanto en contextos nacionales como internacionales. En este sentido, la creciente complejidad de las relaciones internacionales ha facilitado que el listado de actores interesados en el diseño de las cuestiones internacionales se amplíe y se generen contradicciones de naturaleza teórica y práctica. Es muy complejo saber quién y cómo tiene realmente el poder en la esfera internacional en la actual sociedad en red. Las compañías multinacionales, las ciudades, las naciones sin estado, las organizaciones sociales, los movimientos sociales, los individuos o las empresas tecnológicas actúan sin responder ante ningún gobierno capacitado, a menudo sin la rendición de cuentas propias de los Estados democráticos. Su acción se orienta hacia la movilización de individuos en proyectos y actividades entre personas (Payne, 2009).

Para Kurbalija (2002) la experiencia diplomática se vincula con la construcción del conocimiento: diseminación de información, diseño de instituciones y prácticas, así como creación de rutinas de reconocimiento de las partes. Más aún, no hay práctica diplomática sin gestión del conocimiento, sea para conocer las intenciones del 
otro, ordenar las propias ideas, confrontar los distintos informes o comprobar fuentes de información, algo especialmente vital en el actual contexto de la posverdad y las fake-news. Entre las actividades propias de la diplomacia, destaca el manejo de la información y la gestión del conocimiento como instrumentos que moldean la realidad y que dan formato a las prácticas y los usos internacionales. Kiersey \& Neumann (2013:7) consideran que el valor de dicha información internacional depende de la percepción y la capacidad de socialización del profesional diplomático, que está inserto en una constelación de relaciones sociales efímeras. La percepción del otro y la recepción de sus inquietudes y demandas están en la agenda diaria del trabajo diplomático.

La diplomacia es una forma de conocimiento y de revelación del complejo entramado de las relaciones internacionales, a golpe de crisis. Estas crisis se repiten a escala macro y micro, de modo que los acontecimientos se acaban pareciendo unos a otros (Bjola \& Holmes, 2015). Aquí la función de aprendizaje e imitación de comportamientos se alimenta de los medios de comunicación, de las declaraciones políticas y, en los últimos diez años, del uso intensivo de dispositivos tecnológicos. La revelación crea condiciones epistémicas para la comunidad internacional que aprende a crear nuevos mapas cognitivos, que pueden ser maleables por culpa de la desinformación en el actual contexto de la posverdad digital.

\section{Metodología: material y métodos}

La metáfora de Deutsch (1963) que entiende que la comunicación pública suponen hoy en día los nervios es hoy el último refinamiento de la diplomacia, que ha integrado la comunicación política internacional, la diplomacia pública y la digital. Así, esta investigación pretende averiguar cómo opera la neurociencia en el ámbito de la diplomacia pública, acción de comunicación política internacional destinada a la creación de confianza, el establecimiento de relaciones, el diseño de mapa cognitivos y la aprobación de comportamientos.

En este contexto, se pueden adoptar dos enfoques en la aproximación al estudio de las redes sociales. Por un lado se pueden estudiar las diferencias entre el comportamiento social en línea (digital) y fuera de las redes (analógico), y por otro, los comportamientos únicos en el entorno en línea (Meshi, Tamir \& Heekeren, 2015: 771-782).

La neurociencia puede ayudar tanto a construir teorías más robustas de la agencia. En primer lugar, los nuevos conocimientos sobre la intersubjetividad sugieren que los individuos dentro de una interacción social crean un circuito compartido material, es decir, neuronal, que crea una conexión única y estable entre las personas. Esta nueva visión de la intersubjetividad, donde las relaciones están enraizadas en lo material más que en las ideas, tiene profundas ramificaciones para la teoría relacional, que privilegia estas conexiones en la explicación de los resultados. En segundo lugar, el circuito compartido sugiere además que las prácticas de los individuos involucrados en una interacción social no se realizan de manera autónoma, donde cada actor es un agente único y separado, sino que la acción puede ser considerada como un esfuerzo conjunto de varios agentes (Holmes, 2012). La confianza puede ser desarrollada entre los líderes individuales, y luego se va incrustado en sus respectivos gobiernos, burocracias y sociedades, según Wheeler (2015). 
Este trabajo parte de la hipótesis ligada al contexto. Los orígenes de este fin de ciclo de la actual sociedad mediática tradicional tienen que ver con las innovaciones, la tecnología, las formas de dirección o gestión corporativa o con la creatividad, pero tienen sobre todo tienen que ver con "fundamentos conceptuales y morales, con la incapacidad de las mentes habituadas a una sociedad de masas en adecuarse y adaptarse a la velocidad exigida por otros componentes" y con "una situación nueva de sociedad postmediática o postmasas" (Timoteo, 2007: 358). El problema aquí de falta de adaptación, de pérdida de utilidad comunicativa de los medios y fórmulas convencionales (medios, publicidad, marketing, comunicación) está relacionada con un planteamiento anticuado de los fundamentos teóricos del proceso de comunicación, con erróneas perspectivas sobre las formas en que el cerebro humano accede y selecciona la información para proceder a opciones y decisiones, con erróneos enfoques sobre cómo tienen lugar en nuestros días el proceso de construcción de opinión y sus variaciones (Timoteo, 2007: 358).

Este estudio tiene como finalidad estudiar la estrategia digital y multimedia de la comunicación política internacional en Twitter, para conocer mejor el complejo entramado comunicativo en el actual mundo globalizado. Así, en este trabajo aplicamos a la red social Twitter un modelo de análisis del discurso político y mediático desarrollado por Gallardo-Paúls (2016b: 86-147), que permite hacer compatible el estudio de los datos discursivos con propuestas explicativas surgidas a propósito de la neurocomunicación aplicada al ámbito comunicación política y de la comunicación digital. Conforme a la teoría del framing, se parte del principio de que hay categorías del encuadre discursivo que pueden ser tratadas como indicadores de habilidades cognitivas y comunicativas. Desde el análisis crítico del discurso, el acceso al discurso es un elemento básico para la reproducción discursiva del poder (Van Dijk, 2009: 123).

De este modo, se analizan estas categorías agrupándolas en tres dimensiones fundamentales: la intencional (ilocutividad del tuit, encuadre interpretativo de las etiquetas), referencial (temas, protagonistas), e interactiva (alineamiento estructural, predictibilidad; marcas de intertextualidad y dialogismo; afiliación partidista). Conforme a los trabajos de Gallardo-Paúls (2013 y 2014) y Gallardo-Paúls y Enguix-Oliver (2014 \& 2016) el punto de partida de este trabajo es la concepción pragmática del frame o encuadre discursivo, entendido como una noción que el sujeto usuario de las redes sociales, en este caso emisor de mensajes en Twitter, manifiesta no solo en la selección léxica, sino en todas las dimensiones propias del acto comunicativo.

De acuerdo a Gallardo-Paúls (2016b: 92), los estudios que tratan de establecer correlaciones entre neurología y política muestran algunos problemas epistemológicos y se suelen fundamentar en cuatro posibles puntos de partida. En primer lugar, destaca el falso isomorfismo mente-cerebro. En segundo lugar, una cosa es analizar la base biológica de los procesos mentales relevantes en la experiencia política (actividad cerebral) y otra diferente es buscar correlatos fijos y estables entre las estructuras cerebrales y esa misma experiencia política. En tercer lugar, no se puede tratar la política como realidad mental diferenciada. Es necesario reafirmar una heterogeneidad en la concepción de la política. Finalmente, en cuarto lugar, la postura política de un ciudadano no puede considerarse un constructo monolítico y constante, pese a que los experimentos basados en la auto-descripción asuman esa identidad como algo estable y predictor del voto. Así, los citados principios invitar a reforzar el enfoque neurocomunicativo atendiendo a su anclaje cognitivo, y no a los aspectos estric- 
tamente neurológicos. Para dar fundamentación neurocomunicativa a un modelo de análisis del discurso es preciso establecer correlaciones entre elementos neurológicos (la red de las neuronas espejo), habilidades cognitivas (capacidad intersubjetiva) y rasgos discursivos conforme a la propuesta de Gallardo-Paúls (2016b: 94).

\begin{tabular}{|l|l|}
\hline Pragmática enunciativa: intencionalidad \\
\hline Ilocutividad & Tipo de acción que realiza cada tuit \\
\hline Clave interpretativa & Etiquetas /Hashtag \\
\hline Pragmática textual: referencialidad \\
\hline Dimensión predicativa & Protagonistas del tuit \\
\hline Dimensión proposicional & Tema/Asunto \\
\hline Pragmática interactiva: alineamiento y afiliación \\
\hline Predictibilidad y alineamiento & $\begin{array}{l}\text { Menciones @ } \\
\text { Marcas de segunda persona } \\
\text { Sintaxis desencadenante }\end{array}$ \\
\hline Intertextualidad & $\begin{array}{l}\text { Retuits } \\
\text { Citas literales } \\
\text { Enlaces }\end{array}$ \\
\hline Afiliación política & Opinión partidista \\
\hline
\end{tabular}

Tabla 1. Plantilla de metodología de análisis para el estudio por de los indicadores discursivos de subjetividad política en Twitter propuesta por Gallardo-Paúls (2016)

El fenómeno político de Donald Trump, actual presidente de Estados Unidos, tras las elecciones del martes 8 de noviembre de 2016, generó desde el principio el interés de varios investigadores y autores internacionales interesados en su figura. Sin embargo, ninguno de estos estudios se centra en el uso del futuro presidente estadounidense en el uso de las Twitter desde la perspectiva de la neurocomunicación y durante su fase como presidente electo, justo antes de jurar el cargo, fase en la que se pensaba que el nuevo líder político abandonaría planteamientos populistas y demagogos para adoptar una postura más institucional propia de su nuevo rol presidencial. Por eso, este trabajo toma como muestra para su análisis un corpus consistente en un total 134 tuits emitidos por el presidente electo de los Estados Unidos, Donald Trump a través de su cuenta oficial (@realDonaldTrump), en su primer mes como mandatario electo, concretamente entre la noche de su elección el martes 8 de noviembre y el 9 de diciembre de 2016, con el fin de realizar un análisis neurocomunicativo de sus acciones digitales en el ámbito de su acción exterior y diplomática a través de Twitter, convertida en la red social en la que los políticos utilizan para sus comunicados cuando buscan un eco global en la actual sociedad digital. Del análisis de esos datos durante la fase inicial de presidente electo se extraen resultados que corroboran el peligro del mal uso de las redes sociales en la comunicación política en el actual contexto de la posverdad.

\section{Análisis y resultados}

La cuenta oficial de Twitter de Donald Trump, activa desde marzo del año 2009, cuenta con más de 57.820.480 millones de seguidores en enero de 2019. Trump 
contaba 18.164.169 seguidores en diciembre de 2016 nada más ser elegido presidente electo. Trump destaca entre los líderes políticos internacionales. El presidente español, Pedro Sánchez (@sanchezcastejon), a fecha de enero de 2019, no alcanza el millón de seguidores. Se queda en los 965.508. Por su parte, Mariano Rajoy Brey (@MarianoRajoy), mantiene incluso en su actual situación de ex presidente más de millón y medio de seguidores, exactamente 1.619.59.

En el momento de ser votado como presidente, el líder norteamericano seguía tan solo a 41 personas en Twitter. Ahora sigue a 4 más. Entre las personas en las que Trump está interesado se encuentran sobre todos sus familiares, como su actual esposa Melania Knauss-Trump (@MELANIATRUMP) y sus hijos Donald Trump Jr. (@DonaldJTrumpJr), Ivanka (@IvankaTrump),Eric (@EricTrump)y Tiffany (@ TiffanyATrump) y otros familiares como Lara Trump (@LaraLeaTrump) o Vanessa Trump (@MrsVanessaTrump). Un dato importante es que entre los 41 perfiles seguidos por Trump destacan los perfiles de sus negocios más importantes, entre los que destacan las cuentas de los hoteles y resorts Trump en Chicago, Los Ángeles, Washington DC, Las Vegas, National Doce de Miami, Charlotte o de Waikiki en la isla de Oahu en Hawai.

\begin{tabular}{|l|c|c|}
\hline Categoría & Total & Porcentaje \\
\hline Política & 5 & $12,19 \%$ \\
\hline Familia & 7 & $17,07 \%$ \\
\hline Negocios & 11 & $26,82 \%$ \\
\hline Medios de comunicación & 14 & $34,14 \%$ \\
\hline Otros & 4 & $9,75 \%$ \\
\hline & 41 & $100 \%$ \\
\hline
\end{tabular}

Tabla 2. Cuentas de Twitter seguidas por Donald Trump en su elección (Noviembre de 2016)

Destaca como gran colectivo de interés por parte de Trump el de los periodistas y líderes mediáticos. Se trata de un total de 14 profesionales de la comunicación, mayoritariamente de medios de comunicación conservadores, entre las que destacan los periodistas de la cadena de televisión Fox (propiedad del magnate Rupert Murdoch) como Greta Van Susteren (@greta), Eric Bollin (@ericbollin), Laura Ingraham (@ IngrahamAngle), Sean Hannity (@seanhannity)y Bill O’Reilly (@oreillyfactor)y programas como Fox Nation (@foxnation)y Fox and Friends (@foxandfriends). Desde la perspectiva internacional, es importante destacar que solo uno de los periodistas seguidor por Trump no es norteamericano, se trata del periodista sensacionalista inglés Piers Morgan (@piersmorgan), ex responsable de News of the Worldy Daily Mirror. Solo hay un periodista de la NBC, Joe Scarborough. Destacan también casos especiales como la del dúo cómico de youtubers 'Diamond and Silk' formado por las afroamericanas Lynnette Hardaway y Rochelle Richardson.

Del análisis del discurso de Donald Trump en sus primer mes de presidente electo, destacan los mensajes de centrados en los anuncios informativos o de revelación de nuevos nombramientos $(25,05 \%$ del total de tuits) en los que destacan los anuncios de los miembros de nuevo ejecutivo como el fichaje del doctor Ben Carson como responsable de Vivienda y Desarrollo Urbano o del general Mattis como nuevo 
secretario de Estado de Defensa. También se enmarcan en esta categoría los anuncios del propio Trump en eventos y su participación en reuniones, actos con seguidores o en entrevistas con los medios de comunicación, especialmente apariciones en televisión. Le siguen en cantidad, los actos de aleccionamiento, que en este caso serían casi más bien de amonestación. Ocupan el 18,79\% del total de tuits de Trump en su primer mes de presidente electo y los utiliza sobre todo para abroncar y atacar a los medios de comunicación contrarios a su candidatura. Así, hay 9 tuits contra el diario The New YorkTimes (@nytimes)y 5 contra la cadena de televisión CNN (@CNN) que apostaron por la candidata demócrata, Hillary Clinton. Finalmente, destacan por cantidad los mensajes de reconocimiento y en este caso también de agradecimiento de Trump (14,28\%) sobre todo a sus electores. Hay también tuits de reconocimiento a víctimas de incendios, accidentes de tráfico o veteranos de guerra, entre otros. También hay mensajes directos de agradecimiento a otros líderes políticos nacionales como Mitt Romney o los hermanos Jeff y George W. Bush.

\begin{tabular}{|c|c|c|}
\hline Tipo de acto ilocutivo & Número & Porcentaje \\
\hline Revelación & 29 & $21,05 \%$ \\
\hline Recomendación & 6 & $4,51 \%$ \\
\hline Aleccionamiento & 25 & $18,79 \%$ \\
\hline Confirmación & 7 & $5,26 \%$ \\
\hline Interrogación & 5 & $3,75 \%$ \\
\hline Interpretación & 10 & $7,51 \%$ \\
\hline Reconocimiento & 19 & $14,28 \%$ \\
\hline Reflexión & 14 & $10,52 \%$ \\
\hline Otros & 8 & $6,01 \%$ \\
\hline & Total $=134$ & $100 \%$ \\
\hline
\end{tabular}

Tabla 3.- Tipos de actos ilocutivos de Donald Trump en Twitter.

En el marco pragmática enunciativa o intencionalidad, Donald Trump usa en su cuenta oficial diferentes etiquetas o hashtags, entre los que destacan \#ThankYouTour2016 (con tres menciones) pero también hay otros citados como \#heroworldchallenge, \#AmericaFirst, \#ThankAVet, \#ElectionNight, \#CorruptHillary, \#votefraud y \#VPeotus, entre otros.

En el campo de la pragmática textual y la referencialidad, Donald Trump, como emisor, es siempre coprotagonista del tuit, pero a veces centra su atención en otros líderes políticos (su adversaria Hillary Clinton, el fallecido Fidel Castro, el ex presidente George W. Bush, el ex candidato Mitt Romney, el nacionalista británico Nigel Farage o su vicepresidente Mike Pence), problemas sociales (incendios, accidentes, desempleo, deslocalización industrial), eventos cercanos (gira de agradecimiento, mítines y reuniones políticas) o miembros de su equipo (Mike Pence, John Kennedy, Ben Carson, Mick Pompeo o Dan Scavino).

En la dimensión proposicional, entre los temas y asuntos tratados, desde la perspectiva territorial, destaca la mención explícita a ciudades y Estados norteamericanos (34 menciones en las que destacan Luisiana, Indiana, Carolina del Norte, Pennsylvania, Michigan, California, New Hampshire, Ohio, Florida, Nueva York, 
Washington DC y Virginia) frente a 10 menciones internacionales (México, China y Taiwán, Arabia Saudí, Japón, Rusia, Reino Unido o Cuba).

En la pragmática interactiva, las menciones directas con enlaces insertado a otros perfiles de Twitter son casi todas a su vicepresidente@mikepence (6 menciones), a @nytimes (9 menciones), a @CNN (5 menciones) o a @jeffzeleny (4 menciones). Destacan menciones puntuales al deportista Tiger Woods (@TigerWoods), al británico Nigel Farage (@Nigel_Farage). Además de los medios anteriormente citados, en los tuits de Trump se encuentran enlaces a@FoxNews,@60minutes y@foxandfriends. Del mismo modo, aunque no aparecen sus perfiles directos, sí aparecen marcas de segunda persona en referencia a protagonistas de la actualidad americana como Bill Ford, John Kasich, Chuck Jones, Alex Baldwin, John Glenn, Joe Bowman o Steve Doocy, entre otros.

Respecto a la intertextualidad, en los 133 tuits analizados, solo aparecen tres retuits, dos de su colaborador@DanScavino y uno de su equipo@Transition2017. Trump solo sigue a 41 personas en Twitter, por lo que todos los retuits quedan limitados a unos pocos. En relación a los enlaces que Trump muestra en sus tuits, son muy pocos. Aparecen tres enlaces a la web de acceso para lograr tickets para sus eventos o a páginas de Facebook. Dentro de esa intertextualidad, destacan enlaces a 12 herramientas multimedia (vídeos, presentaciones u otras redes). También hay que destacar que solo 12 tuits $(9 \%)$ de los 133 cuentan con fotografías complementarias del texto. Sobre citas textuales y literales citadas literalmente en los tuits de Trump solo encontramos cinco menciones entrecomilladas o directas a declaraciones o actos de habla de otros como pueden ser titulares de The New York Times o declaraciones de Hillary Clinton. Finalmente, respecto a la filiación política, evidentemente todas giran alrededor de la propia figura de Donald Trump como nuevo líder del Partido Republicano. En ningún momento aparecen citas a los opositores y voces críticas que el propio Trump tiene dentro de su formación.

\section{Discusión y conclusiones}

Las neurociencias se han derivado a otros ámbitos científicos y específicamente a ciencias sociales, incluyendo tanto las ciencias de la comunicación como la ciencia política y sociológica. Así, están llegando a cambiar radicalmente todo lo relativo al acceso de los individuos a la información y al conocimiento en la actual sociedad digital. Hoy en día, el entorno no es una estructura impuesta desde el exterior sino una creación de los propios seres vivos o de cómo el modelo red manifiesta y expresa un pensamiento conectivo gracias al fenómeno de las redes sociales. El análisis de las redes sociales desde el punto de vista de la política internacional es un reto de futuro tanto para la neurocomunicación. El acto comunicativo no es una simple transferencia de mensajes sino una interacción de códigos con factores comunes.

El estudio de la actual sociedad digital y el fenómeno de la posverdad y las fakenews permite entender y describir la llegada al poder Donald Trump, un caso en el que la posverdad ha jugado un papel trascendental en la medida en que ha sobrepasado cualquier expectativa racional predecible por los expertos. De acuerdo con los posicionamientos tradicionales, nuestros cerebros y mentes están moldeados por nuestras experiencias, que tienen lugar principalmente en el contexto de la cultura en la que vivimos y nos hemos formado. En paralelo, los psicólogos no dejan de 
descubrir abundantes evidencias sobre la diversidad de la cognición humana y el comportamiento a través de los diferentes procesos culturales en los que las redes sociales cada vez tienen más peso. En este contexto, la posverdad denota circunstancias en que los hechos objetivos influyen menos en la formación de la opinión pública, como los llamamientos a la emoción y a la creencia personal, influenciados por contenidos digitales.

El estudio concreto del caso de los 133 tuits del presidente Trump durante su primer mes tras ser proclamado vencedor de los comicios de noviembre de 2016 además de ofrecer muchísima información sobre lo que han sido las posteriores líneas de actuación de Trump, confirma los peores presagios sobre el uso que se puede hacer de las redes sociales desde una óptica populista. Donald Trump ha empleado las redes sociales para incrementar la afiliación partidista y manejar la opinión pública. Por un lado, mediante la exposición de ideas no convencionales sobre las relaciones internacionales de Estados Unidos. Esta postura cambia el marco de entendimiento y aliados con tuits concretos sobre Rusia, Arabia Saudí, China y la región de Asia-Pacífico, con la polémica mención a Taiwán. También disminuye el peso de la Unión Europea, institución supranacional de primer orden que no solo es ignorada, es menospreciada con los halagos a su opositor Nigel Farage. Destaca también la mención negativa a la industrialización de México. También destaca el enfrentamiento directo con amonestación explícita de Trump como presidente electo contra The New York Times y la televisión CNN, algo inédito hasta ahora en un jefe de Estado de un país democrático occidental.

Por otro lado, porque el reflejo de los medios de comunicación internacionales refuerza el perfil heterodoxo del nuevo presidente. Ese posicionamiento refuerza la identidad del votante republicano. A través del mensaje corto enviado a la audiencia global a través de Twitter en un principio y repetido después por las grandes compañías de medios audiovisuales, como el canal de televisión de la Fox, se refuerza el mensaje para las audiencias locales o internas de su propio electorado en Estados Unidos. El espectáculo de Trump es el conducción de un yo simbólico, una comunidad imaginada que ha perdido su posición de privilegio ante los avances y retrocesos de la globalización en el actual contexto digital de la posverdad.

\section{Referencias bibliográficas}

Alvídrez, S. y Franco-Rodríguez, O. (2016): "Estilo comunicativo súbito en Twitter: efectos sobre la credibilidad y la participación cívica", Comunicar, 47, XXIV, pp. 89-97. http:// dx.doi.org/10.3916/C47-2016-09

Bjola, C. y Holmes, M. (2015): Digital Diplomacy: Theory and Practice, London, Routledge- New Diplomacy Studies.

Bound, K; Briggs, R; Holden J And Jones, S. (2007): Cultural Diplomacy, London: Demos.

Damasio, A. (1994): Descartes' Error: Emotion, Reason and the Human Brain, London: Pan Macmillan.

Deutsch, K. (1963): The Nerves of Government. Models of Political Communication and Control, New York: The Free Press of Glencoe.

Enguix-Oliver, S. y Gallardo-Paúls, B. (2015b): "Opciones discursivas en la cobertura electoral: los temas en la campaña europeas de 2014”. En López, M. I. y Hernández, E. (Coords.): Sodalicia Dona. Homenaje a Ricardo Escavy Zamora, Universidad de Murcia, pp. 231-152. 
Enguix-Oliver, S. y Gallardo-Paúls, B. (2015a): "Política televisada y su reflejo en Twitter: medios y seguidores", Workshop on Communication and Journalism in Social Networks, 10th Iberian Conference on Information Systems and Technologies (CISTI 2015). Aveiro, 17-20/06/2015.

Gallardo Paúls, B. y Enguix Oliver, S. (2016): Pseudopolítica: el discurso político en las redes sociales, Valencia: Departament de Teoria dels Llenguatges i Ciències de la Comunicació-UV, https://www.researchgate.net/publication/299284420_2016_Pseudopolitica_el_discurso_politico_en_las_redes_sociales

- (2014): "Estrategias de encuadre discursivo en periodismo político: análisis de un corpus de titulares", Círculo de Lingüistica Aplicada a la Comunicación, 58, pp. 90-109.

Gallardo-Paúls, B. (2013): "Niveles pragmáticos y cognición: estrategias discursivas de encuadre en el discurso político", Revista Anthropos, 239, pp. 191-210.

- (2014): Usos políticos del lenguaje. Un discurso paradójico. Barcelona: Anthropos/Siglo XXI.

- (2016a): “¿Neuronas espejo o capacidad intersubjetiva? Referencias viables para un análisis del discurso público de base neurocomunicativa", I Congreso Internacional Comunicación y Pensamiento. Comunicracia y Desarrollo Social, Sevilla, 10-12/03/2016. DOI: 10.13140/RG.2.1.2556.3286

- (2016b): "Programas de tertulia política en Twitter: un modelo neurocomunicativo de análisis del discurso". Círculo de lingüística aplicada a la comunicación, 66, pp. 86-147. http://dx.doi.org/10.5209/CLAC.52770

Han, S. y Northoff, G. (2008): "Culture-sensitive neural substrates of human cognition: a transcultural neuroimaging approach". Nature Reviews Neuroscience 9, pp. 646-654 (August 2008), doi:10.1038/nrn2456

Holmes, M. (2012): The Material of Diplomacy: Neuroscience and Agency in International Theory. ECPR Joint Sessions, University of Antwerp (Belgium), 10-15 April 2012. https://ecpr.eu/Events/PaperDetails.aspx?PaperID $=5897 \mathrm{yEventID=6}$

Kiersey, N. J. y Neumann, I. B. [Coord.] (2013): Battlestar Galactica and international relations Popular culture and world politics, London: Routledge.

Kurbalija, J. (2002): Knowledge and Diplomacy, Brussels: DiploPublishing.

Lakoff, G. (2009): The Political Mind: A Cognitive Sientist's Guide to Your Brain and Its Politics. London: Penguin.

Macías-Varela, B. (2017): La comunicación para el cambio social desde una perspectiva de género en Andalucía. Tesis doctoral. Universidad de Cádiz.

Meshi, D., Tamir, D. y Heekeren, H. R. (2015): “The Emerging Neuroscience of Social Media". Trends in Cognitive Science, 19, 12, pp. 771-782 (December 2015) http://dx.doi. org/10.1016/j.tics.2015.09.004

Payne, J.G. (2009): "Trends in Global Public Relations and Grassroots Diplomacy", American Behavioral Scientist, 53, 4, pp. 487-492.

Quintas-Froufe, N. y Neira González, A. (2014): “Audiencias activas: Participación de la audiencia social en la televisión”, Comunicar, 43, XXII, pp. 83-90. http://dx.doi. org/10.3916/C43-2014-08

Rodríguez-Ferrándiz, R. (2017): Máscaras de la mentira: el nuevo desorden de las posverdad. Pretextos-Ajuntament de València/Ayuntamiento de Valencia.

Rubio-Moraga, Á. y Sapag-Muñoz De La Peña, P. (2014): "Neuropolítica". En Timoteo, J. (Coord.): Neurocomunizacione, Roma: Aracne, pp. 185-220. 
Schreiber, D. y Iacoboni, M. (2012): "Huxtable on the brain: An fMRI Study of Race and Norm Violation". Political Psycology, 33, pp. 313-330. http://dx.doi.org/10.1111/j.14679221.2012.00879.x

Schreiber, D; Fonzo, G., Simmons A. N. et al. (2013): Red Brain, Blue Brain: Evaluative Processes Differ in Democrats and Republicans. PLoS ONE 8(2): e52970. doi:10.1371/ journal.pone.0052970

Timoteo, J. (2005): Gestión del poder diluido. Madrid. Pearson.

- (2007): "Neurocomunicación. Propuestas para una revisión de los fundamentos teóricos de la comunicación y sus aplicaciones industriales y sociales". Mediaciones Sociales, 1, pp. 355-386.

- (2014): "Il fattore 'Pentola a induzione' nei procesi di Intelligenza Connettiva". En Timoteo Álvarez, J. (Coord.): Neurocomunicazione, Roma: Aracne,. pp. 43-68.

Uña Juárez, O.; Fernández- Fernández, M. Yy Fernández-Camacho, C. (2014): “De tutti e di nessuno. Struttura dei processi nella creazione dell'opinione pubblica". En Timoteo Álvarez, J. (Coord.): Neurocomunicazione, Roma: Aracne, pp. 113-142.

Van Dijk, T. (2009): Discurso y poder, Barcelona: Gedisa.

Wheeler, N. J. (2015) 'Face-to-face Diplomacy and Interpersonal Trust in International Relations'. University of Birmingham (May 2015). http://www.birmingham.ac.uk/schools/ government-society/centres/iccs/news/2015/05/Workshop-report-Face-to-face-Diplomacy-and-Interpersonal-Trust-in-IR.aspx 